\title{
DESIGN OF THE VLPC CRYOSTATS
}

\author{
D-ZERO ENGINEERING NOTE \# 3823.113-EN-559
}

July 19, 2001

Author: Russell A. Rucinski

PPD/MSD/D0 Operations

This paper was written for the Cryogenic Engineering Conference. To be published in Advances in Cryogenic Engineering, volume 47 or 48. 


\title{
SHORT PROFILE RECTANGULAR HELIUM CRYOSTAT MADE FROM NICKEL-IRON ALLOY*
}

\author{
Russell A. Rucinski \\ Fermi National Accelerator Laboratory \\ P.O. Box 500 \\ Batavia, IL 60510, USA
}

\begin{abstract}
A short profile $(40 \mathrm{~cm})$, long length $(2.50 \mathrm{~m})$ rectangular helium cryostat has been designed, built and tested for maintaining electronic chips at a stable 9.0 Kelvin temperature. Heat load, and thermal stresses were major design considerations. The distance from room temperature to liquid nitrogen intercept temperature is only 8 centimeters. Liquid nitrogen temperature to liquid helium temperature occurs in 13 centimeters. An alloy known as Invar, 36\% Nickel, balance Iron, was used to fabricate the vessel walls and liquid nitrogen heat intercept tubing because of it's low coefficient of thermal expansion. The cryostat accepts fifty-one, $3.5 \mathrm{~cm} \mathrm{x} 42.5 \mathrm{~cm}$ devices that extend $26.7 \mathrm{~cm}$ into the cryostat. Tight clearance fits to the liquid nitrogen and liquid helium intercepts are maintained. Gaseous helium is used within the cryostat for heat transfer.
\end{abstract}

\section{INTRODUCTION}

A special purpose cryostat has been designed and built at Fermi National Accelerator Laboratory for cooling electronic chips to an operating temperature of approximately $9.0 \mathrm{~K}$ $+/-0.1 \mathrm{~K}$. The electronic chips are known in the physics community as visible light photon counter (VLPC) chips. They are used for readout of scintillating fiber tracker barrels that surround the proton/anti-proton collision point in the D-Zero detector. A total of two symmetrical special purpose cryostats were made.

Competing design constraints made for an interesting design challenge. Approximately 50,000 optical fibers, 835 microns in diameter feed into each cryostat in a dense layout. At the cold end of the cryostat, VLPC chips convert light signals into electronic signals. Signal cables run from the cold end back up to the warm end and out of the cryostat to amplifier boards. Because the electronic signal generated by the chips is small, electrical designers wanted to maximize the copper cross sections for the cables and minimize the

* Work supported by the U.S. Department of Energy under contract No. DE-AC02-76CHO3000. 
length between the VLPC chip (cold end) and the amplifier boards (warm end). Thus one of the major constraints was a short profile. Short distances in cryogenics translate into large heat loads, and thermal stress concerns. The dense layout required a rectangular shaped vessel. Rectangular shapes (versus cylindrical) for pressure vessels mean concern about stress and deflection from pressure and corner joints.

After presenting the general description of the cryostat, this paper will discuss specific design requirements and the choices made to satisfy them.

\section{DESCRIPTION OF CRYOSTAT CONSTRUCTION}

An assembly drawing with sectional views and labels of fundamental parts is shown in Figure 1. I will discuss the parts starting at the room temperature top lid and working towards the cold end.

There are 51 slots to accept $3.5 \mathrm{~cm}$ wide $x 42.5 \mathrm{~cm}$ long x $26.7 \mathrm{~cm}$ long VLPC cassette packages in the top lid. The slot size and location tolerances were held to $0.13 \mathrm{~mm}$. The flatness and surface finish of the lid was controlled to enable molded polyurethane gaskets to seal around the perimeter of each slot opening. The top lid was machined flat from a 3.5 $\mathrm{cm}$ thick type 304 stainless steel plate to a final thickness of $2.8 \mathrm{~cm}$. Two $12 \mathrm{Watt} / \mathrm{cm}$ cable heaters are epoxied into a $3.3 \mathrm{~mm}$ radial groove that encircles the slots.

The inner box of the cryostat is constructed of $36 \%$ nickel, balance iron alloy known as Invar 36 . The walls of the box are $3.2 \mathrm{~mm}$ thick. 52 rectangular invar tubes span across the long walls on each side of a cassette location. The tubes are precisely located relative to the lid slots and set $0.4 \mathrm{~mm}$ of clearance between the tube and the cassettes that are inserted into the slots. During operation, the tubes fill with liquid nitrogen supplied by an invar cooling channel that is welded integral on the outside of the invar side walls. A little further down, $8 \mathrm{~mm}$ diameter invar stay rods span across the long walls to react against the forces of $0.18 \mathrm{Mpa}$ ( $3 \mathrm{psig}$ ) gas helium pressure internally and vacuum on the exterior. Stiffening rails provide strength on the short walls. The bottom of the inner box is the least interesting part, $1.27 \mathrm{~cm}$ thick with some leveling screws.

A high purity copper cold block sits upon the leveling screws and serves as the heat sink for the cold end of the VLPC cassettes. Liquid helium flows through pipes soldered to the cold block. High purity copper "T" shaped cross pieces sit upon the top of the cold block. The top surface of the cold block and the bottom surface of the "T"' cross pieces were machined flat to $0.03 \mathrm{~mm}$ over the $188 \mathrm{~cm}^{2}$ contact area. The pocket formed by adjacent "T' cross pieces give $0.8 \mathrm{~mm}$ clearance around the cold end of the cassette.

Rohacell foam lines the interior perimeter of the box to prevent convection currents. A copper radiation shield and multi-layer insulation are located in the vacuum space and surround the outside of the cassette space box. The insulating vacuum vessel is made of 304 stainless steel plates that are welded to the top lid.

\section{DESIGN REQUIREMENTS AND CHOICES}

\section{Invar 36 material for inner vessel}

The elevation of the VLPC cassette's liquid nitrogen intercept was pushed as close to the warm end as possible to maximize the thermal distance below, between the liquid nitrogen intercept and cold block. This imposed a large, $35 \mathrm{~K} / \mathrm{cm}$ thermal gradient from the lid to the nitrogen cooled level. The dense layout also required that the cryostat have a 

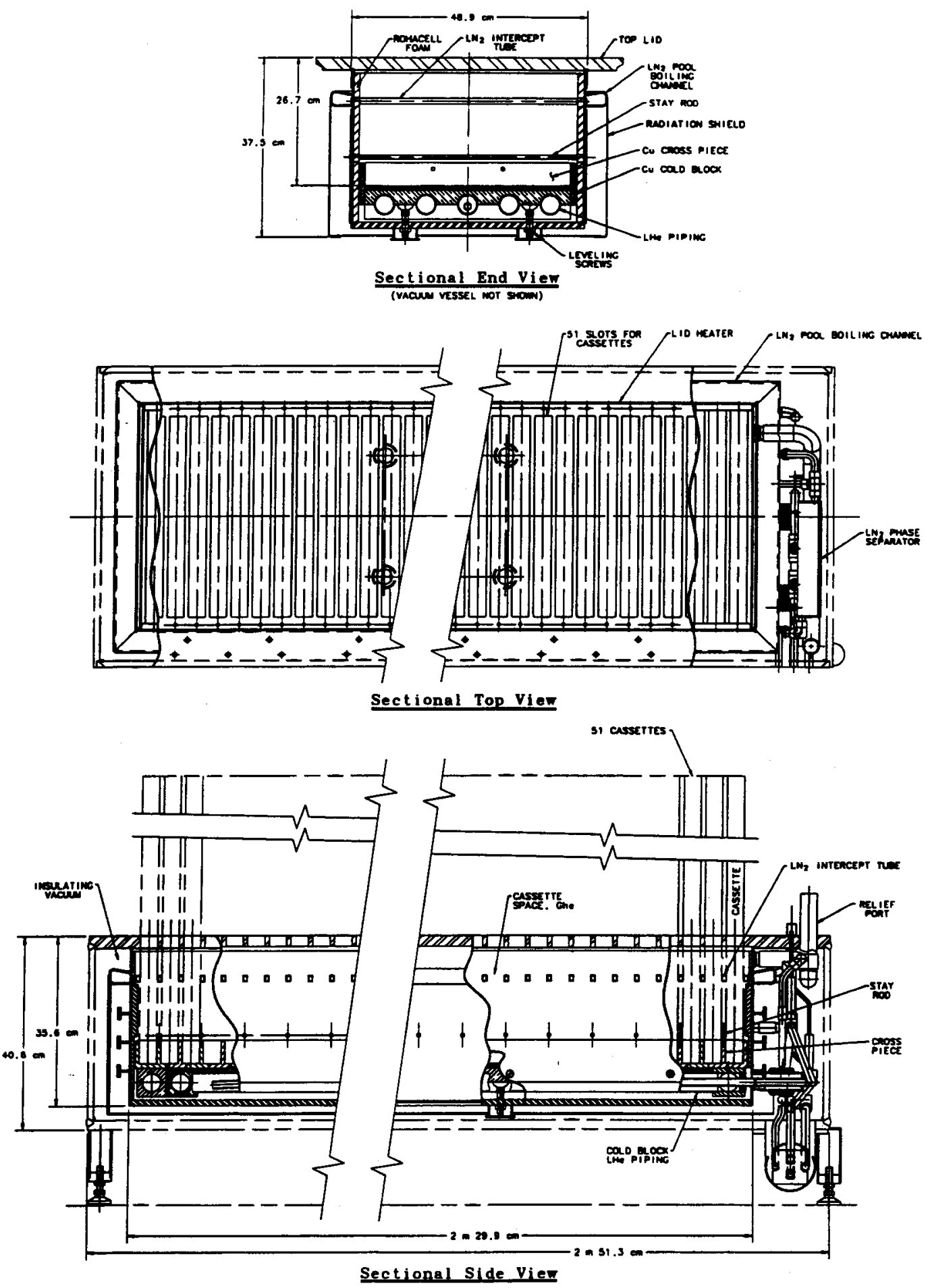

FIGURE 1. Sectional views of the cryostat and nomenclature for fundamental parts. 
long $2.3 \mathrm{~m}$ continuous wall. Invar 36 was selected as the inner box material primarily for it's very low coefficient of thermal expansion. Linear thermal expansion from room temperature to liquid nitrogen temperature is $-0.00040 \mathrm{~m} / \mathrm{m}$ for invar versus $0.0029 \mathrm{~m} / \mathrm{m}$ for type 304 stainless steel. The linear length at the intercept decreased $0.9 \mathrm{~mm}$ for invar versus $6.7 \mathrm{~mm}$ for stainless steel. The shear strain in the upper corners of the long side plate would be well above yield for any other material than invar. Even using invar, the stresses reached about $200 \mathrm{MPa}$ (roughly $75 \%$ of yield) at the upper corners where the plate is welded to and constrained by the top lid.

The choice of invar also aided in keeping the close gap alignment of the VLPC cassette body intercepts to the nitrogen intercept $(0.4 \mathrm{~mm})$. The cost of invar ranged from $\$ 35$ per kilogram for plate, to $\$ 100$ per kilogram for filler rod. Invar was welded to the type 304 stainless steel lid using invar filler rod and the GTAW process. A welding procedure specification and welders were qualified by an independent testing laboratory as conforming to Section IX of the ASME boiler and pressure vessel code. Invar material was not listed in the ASME boiler and pressure vessel code but code rules were followed to the extent possible. Allowable stresses were determined for individual components by applying ASME (Section II, part D, appendix 2) philosophy using yield and ultimate stress values listed on the material certification reports or by actual tension testing results. The design followed ASME Section VIII, Div. 2 rules. Both hand calculations and finite element analysis were used to determine stress levels and optimize the design.

Special attention was paid at piping attachments to or through the wall. In all cases a material transition from invar to stainless steel occurred. A thin wall invar coupling was welded to the wall and then welded to the stainless piping about $25 \mathrm{~mm}$ away from the wall. Had the stainless tubing been welded directly to the wall, calculations showed that unacceptably high tangential stresses would have occurred when the parts got cold. The stainless tube shrinks whereas the invar plate undergoes very little dimensional change. Using a transition coupling about the same strength as the stainless tube allowed stresses to be shared equally in both the coupling and tubing with the stress levels being $227 \mathrm{Mpa}$ in each part.

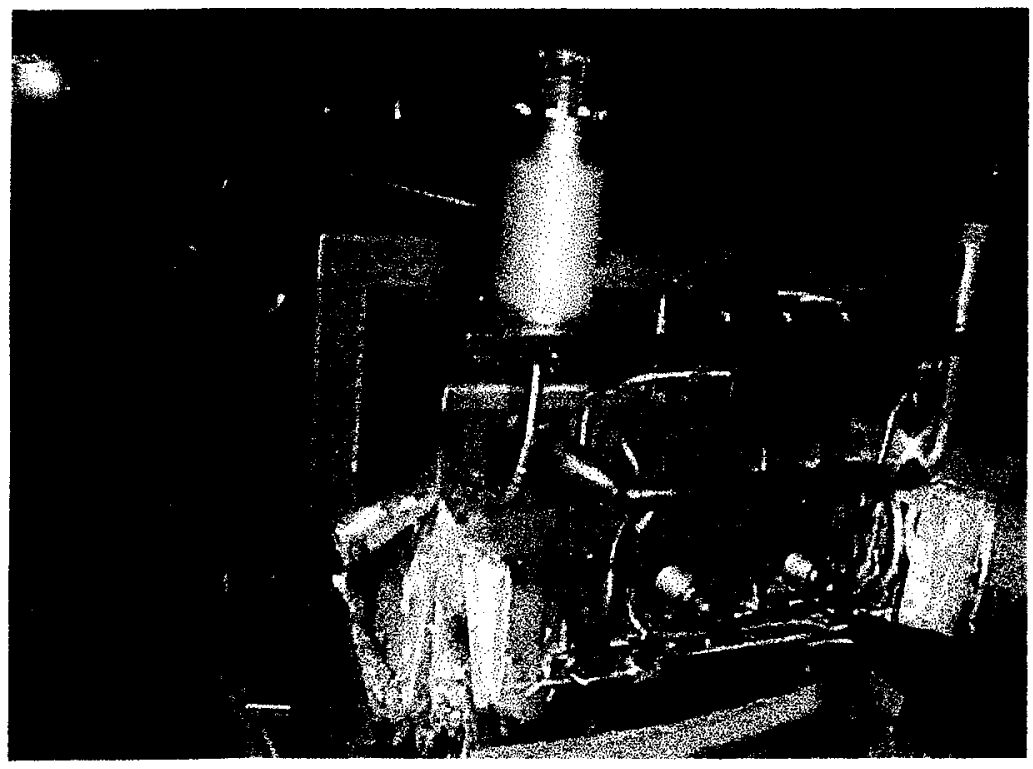

Figure 2. Piping end of cryostat without vacuum jacket. Instrumentation port is on left, relief valve port is on the right. The helium supply and returns exit the invar box through bellows at the bottom. Copper radiation shield hangs from liquid nitrogen cooling reservoir channel along long side. 


\section{Liquid nitrogen intercept}

The upper nitrogen intercept rectangular tubes accomplished several design needs. First and primarily they served as a heat intercept for the cassette. Good alignment of the liquid nitrogen intercept tubes to the slots in the top lid was accomplished by using a tooling jig during welding of the tubes to the walls. Each $(12.7 \mathrm{~mm}$ high $\times 8.9 \mathrm{~mm}$ wide $\times 0.9 \mathrm{~mm}$ wall) tube absorbs 8 watts of heat across the gas gap from adjacent cassettes. The temperature of the cassette at the intercept station is effectively lowered to less than $95 \mathrm{~K}$. Both conduction heat load coming down the copper traces of the electrical signal cables and radiation heat from the lid are intercepted and absorbed into the liquid nitrogen tubes. The tubes are kept flooded with liquid nitrogen by using a pool boiling design. The tubes extend into a reservoir that rings the invar box. The reservoir is topped off with liquid by a phase separated liquid nitrogen supply. Gas generated in the rectangular tubing rises by buoyant forces into the top portion of the reservoir and out a gas nitrogen pipe. Heat load down the invar side wall is also efficiently absorbed into the liquid of the reservoir. The rectangular tubes also act as tension members between the adjacent long walls to resist the $0.12 \mathrm{Mpa}$ pressure differential across the wall. This allowed the side wall thickness to be minimized to $3.2 \mathrm{~mm}$, the minimum thickness allowed by ASME VIII-2 rules. Figure 3 is a photograph showing the intercept tubes and reservoir during assembly. Closer to the cold end, a row of solid $8 \mathrm{~mm}$ diameter rods also span across the longer walls as stays against pressure. The drawn rectangular tubes, formed reservoir channel, and solid stay rods are all invar material that were heli-arc welded to the side wall using invar filler rod.

The design heat loads for the liquid nitrogen intercept were 650 watts from the wall, 400 watts intercepted from the cassettes, and 135 watts intercepted from gas helium/foam in the cassette space. The design liquid nitrogen flow rate for the cryostat was about 35 liters/hour $(9 \mathrm{gph})$. In real operation, we have found that it is necessary to flow five times that amount to get sufficient flooding of the reservoir. I believe that had the liquid supply tubing size that feeds the reservoir been larger, the required flow would have been closer to design values. It is a supply problem rather than an increased heat load problem.

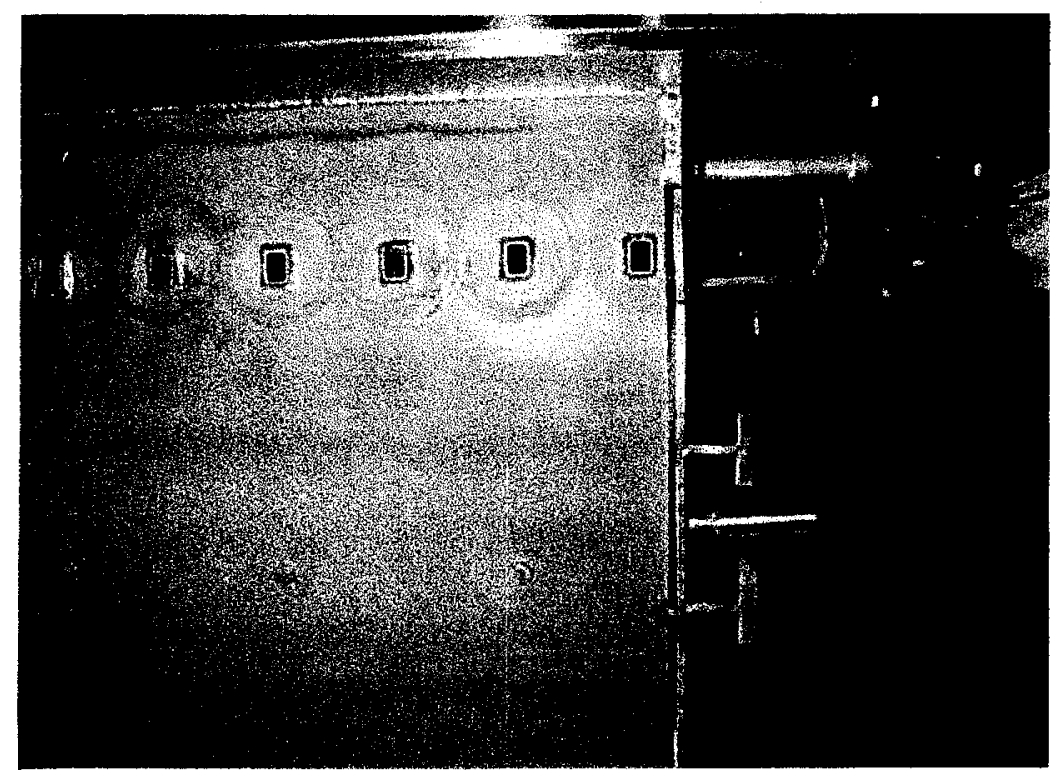

FIGURE 3. Rectangular tubing for the liquid nitrogen intercept before being covered by liquid reservoir channel (seen on end wall on right). The ends of the solid stay rods can be seen lower down on wall. 


\section{Copper cold block}

Alignment of the slots/cassette to the cold end copper heat sink pockets was assured by allowing the copper heat sink pockets to move. The inverted " $T$ " shapes that created the close fitting ( $0.8 \mathrm{~mm}$ clearance) pockets were finished flat and smooth on the bottom so that they could slide on the copper cold block base. The copper cold block base was made by oven soldering ( $96 \% \mathrm{Sn}, 4 \% \mathrm{Ag}$ solder) high purity copper (UNS C10100) half blocks on top of five $38 \mathrm{~mm}$ diameter x $2.2 \mathrm{~m}$ long copper tubes. After soldering, the top surface of the copper half blocks was machined smooth and flat also. See Figure 4 for a photograph of the cold block assembly as it looked before being welded into the invar box. The cold block is actively cooled to a temperature of around $6.0 \mathrm{~K}$ by flowing $7.5 \mathrm{~g} / \mathrm{s}$ of liquid helium through the five tubes. The cold block is pinned to the invar box at it's center. It is allowed to shrink $(7.7 \mathrm{~mm})$ towards the center while the cassettes hold the inverted "T"'s stationary. Two stainless steel $0.79 \mathrm{~mm}$ diameter wire clips were placed over the upward leg of the cross pieces and act like bumpers against the cassette. This gives a uniform side gap and uniform free convection heat transfer from the cold end of the cassette to the cross piece. The cold block assembly depth from the lid was adjusted with leveling screws after all the welding was done to the cassette space box.

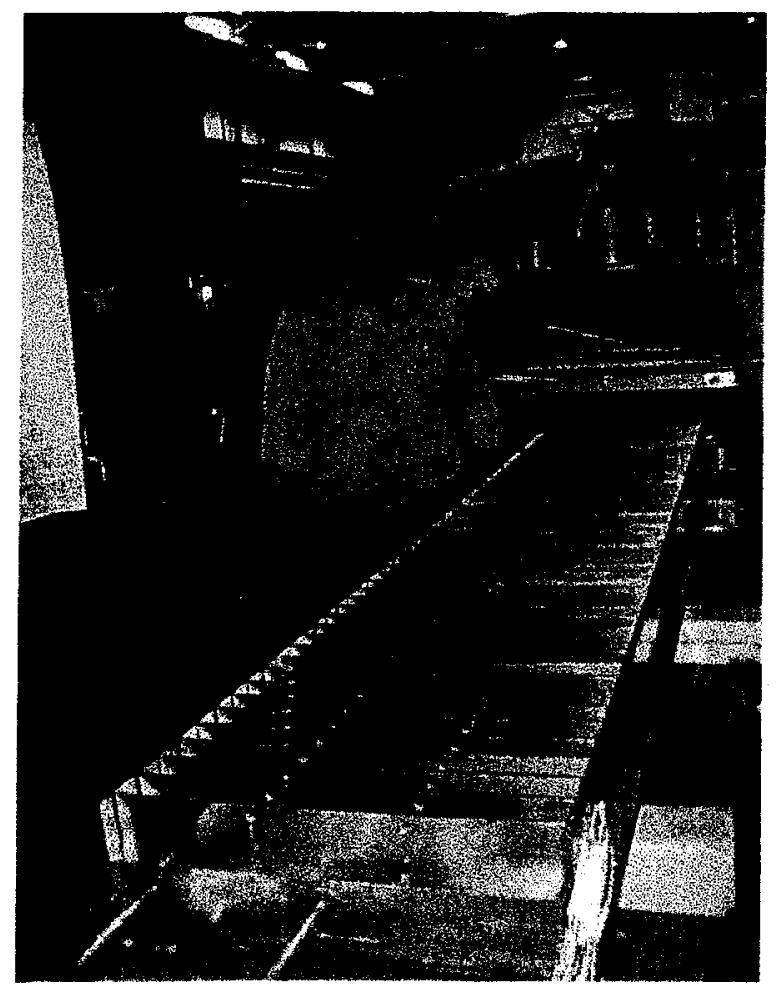

FIGURE 4. Copper cold block assembly ready to be inserted into cryostat.

Stable temperature control of the cold block to $+/-0.1 \mathrm{~K}$ was desired. One feature to achieve this was to size the cold block cooling tube volume to be around 3 times the volume of the liquid helium supply transfer line. This was expected to buffer out any transients in the supply fluid quality. Another feature used was that the supply line heat 
exchanged with the fluid resident in the middle cold block pipe. A third feature was that at the design point, liquid helium lies in the bottom of the cold block pipes in a very low laminar flow state. Raising or lowering the liquid level cools or warms the gross temperature of the block. The flow control valve for the helium is on the exit piping from cold block where it is assured that the fluid is in the gas phase.

The design goals for the cold block were met. The cold block temperature controls to within $0.1 \mathrm{~K}$. The cold block can be reduced to run as cold as $5.0 \mathrm{~K}$. The operating point that best achieves $9.0 \mathrm{~K}$ for the cassettes is at a temperature of $6.2 \mathrm{~K}$. The overall heat load to the liquid helium coolant has been measured and agrees with the expected design numbers. Specifically, 50 watts come from the cryostat walls and 90 watts come from the 51 cassettes.

\section{Cost and content comments}

Each cryostat cost about $\$ 100,000$ and 7 months to build. The cost of the cryostat however, pales in comparison to the worth of the VLPC cassette packages that are inserted inside. The VLPC cassettes were uniquely built at Fermi National Accelerator laboratory over several years and have an estimated worth of $\$ 62,500$ each or $\$ 3,000,000$ per cryostat load. At that level of worth, content protection was well scrutinized.

Redundancy was used on critical devices and automatic interlocks were incorporated to automatically keep the cryostat and its contents safe. Measures are taken to ensure that the cassette space is always kept at a positive pressure. This ensures that any leaks will be leaks of helium out rather than air or condensation in, where it could freeze and damage miniature wire bonds or pixels on the VLPC chips.

The lid is required to be heated with about 1200 watts of electrical power to keep the polyurethane gaskets seals of the cassettes at room temperature. Software and hardwired protection keep the heaters from being able to overheat the cassettes. If heater power fails, the cryogenic supply valves close and the lid temperature stays above freezing. 